\title{
NUMERICAL INVESTIGATION OF FORCED CONVECTIVE HEAT TRANSFER AROUND A SOLID CIRCULAR CYLINDER UTILIZING NANOFLUID IN UNSTEADY REGIME
}

\author{
Yacine Khelili $^{1^{*}}$ - Abderrazak Allali ${ }^{1}$ - Rafik Bouakkaz ${ }^{2}$
}

${ }^{1}$ Aircraft Laboratory, Department of Mechanical Engineering, Univ. Blida 1, Algeria

${ }^{2}$ Department of Mechanical Engineering, Univ. Constantine 1, Algeria

\begin{tabular}{l}
\hline ARTICLE INFO \\
\hline Article history: \\
Received: 25.5 .2017$. \\
Received in revised form: 13.9 .2017$. \\
Accepted: 10.10 .2017$. \\
\hline Keywords: \\
Unsteady flow \\
Nanofluid \\
Volume fraction \\
Finite volume \\
Reynolds number \\
Circular cylinder \\
\hline
\end{tabular}

DOI: http://doi.org/10.30765/er.39.3.07

\section{Introduction}

The classical problem of viscous incompressible flow over a circular cylinder confined in a channel is one of the most widely studied problems in computational fluid dynamics (CFD). This type of flow problems frequently arise in various engineering fields. Because of its popularity, a plethora of numerical, theoretical and experimental results are available for this problem in the literature.

For a certain range of Reynolds numbers, vortex shedding is observed with the pattern of alternating two-dimensional counter-rotating vortices convecting downstream representing the Kármán

\begin{abstract}
:
This paper presents a numerical solution for low Reynolds number, unsteady flow around, and heat transfer from a stationary circular cylinder placed in a uniform flow. The fluid is assumed to be incompressible and of constant property. Twodimensional and unsteady mass continuity, momentum, and energy equations have been discretized using finite volume method. A SIMPLE algorithm has been applied for solving the pressure linked equations. The range of Reynolds numbers was investigated which varied from 50 to 300 with volume fraction on $\mathrm{Cu}$ nanoparticles varying from 1 to $5 \%$ at the constant wall temperature. The results of the heat transfer characteristics of nanofluid flow over a circular cylinder showed marked improvement comparing with the base fluid. It is found that the vorticity, pressure coefficient, and recirculation length are increased by the addition of nanoparticles into base fluid. Moreover, the local and mean Nusselt numbers are enhanced due to adding nanoparticles into base fluid.
\end{abstract}

vortex street (after von Kármán (1911) who studied the stability of the arrangement of the vortices in the wake). In the range of Reynolds numbers between $40<\operatorname{Re}<10000$, Roshko A. [1] observed different regimes: a stable range characterised by laminar vortex shedding for $40<\operatorname{Re}<150$; a transition regime in the range $150<\operatorname{Re}<300$ with irregular bursts of the velocity in the wake and an irregular regime for $300<\operatorname{Re}<10000$ where transition occurs in the separated shear layers.

Some of the notable works on flow past a circular cylinder by the conventional numerical methods include those of [2] Rajani B. N. et al, in this study, we have four regimes of flow: creeping laminar state

\footnotetext{
* Corresponding author.

E-mail address: khliliyacine1@gmail.com.
} 
(L1) of flow $(0<\operatorname{Re}<4)$; laminar flow (L2) with steady separation $(4<\mathrm{Re}<48)$ forming a symmetric contrarotating pair of vortices in the near wake; laminar flow (L3) with periodic vortex shedding $(48<\operatorname{Re}<180)$ and finally, (L4) part of the transitionin-wake $(\mathrm{TrW})$ regime $(180<\mathrm{Re}<400)$ when the three- dimensional instabilities lead to the formation of streamwise vortex structure. Norberg C. [3] has reported the critical Reynolds number for the onset of vortex shedding to almost be constant at $\mathrm{Re}=47.4$, but only for cylinders with aspect ratio larger than 40 . This onset may be delayed as the aspect ratio decreases.

Mahfouz and Badr [4] carried out a numerical study on a fixed and a rotationally oscillating cylinder between $\mathrm{Re}=40$ and 200. They attempted to investigate the possibility of controlling heat transfer using a rotationally oscillating cylinder. Taneda S. [5], investigated flow past a rotationally oscillating cylinder at $(30<\operatorname{Re}<300)$ by means of flow visualization, and found that vortex shedding completely disappears at a very high forcing frequency. Williamson [6] show the Strouhal number for flow over a single cylinder as the aspect ratio (AR) is increased. He showed that the Strouhal number is around 0.14 for aspect ratio so of 30 and below and around 0.16 for higher aspect ratios.

By using different methods, such as expanding the effective heat transfer area or heat transfer coefficient, convective heat transfer can be moderated. Recently, a modern class of fluid, called nanofluid, has been developed and is used to enhance the heat transfer.

The study of convective transport of nanofluids has gained considerable importance due to its applications. Most of the conventional transfer fluids like oil, water, and ethylene glycol have poor heat transfluids because the thermal conductivity of these fluids plays an important role on the heat transfer coefficient between the heat transfer medium and the heat transfer surface. Various numerical and experimental studies of the importance of these nanofluids has been studied and concluded in the literature.

Experimental results [7-10] have illustrated that the thermal conductivity of nanofluid can be increased between $10-50 \%$ via introduction of a small volume fraction of nanoparticles.

Dalkilic et al. [11] has reported critical information on theoretical, experimental, and numerical work related to forced convection heat transfer of nanofluids. Sarkar et al. [12] investigated numerically mixed convective heat transfer for non-
Newtonian nano-fluids around a square cylinder in a vertical flow configuration. They presented the effects of Re, Ri and $\varphi$ numbers on heat transfer. The momentum and forced convection heat transfer for a laminar and steady free stream flow of nanofluids past an isolated square cylinder have been studied numerically by Farooji et al. [13].

Another numerical study on fluid flow and heat transfer around a solid circular cylinder utilizing nanofluid was done by Valipour and ZareGhadi [14]. Their results showed that as the solid volume fraction increases, the magnitude of minimum velocity in the wake region and recirculation length increases while separation angle decreases. Valipouret et al. [15] investigated a numerical simulation to study the fluid flow and heat transfer around a square cylinder utilizing $\mathrm{Al}_{2} \mathrm{O}_{3}-\mathrm{H}_{2} \mathrm{O}$ nanofluid for low Reynolds numbers. The Reynolds number varied within the range of 1 to 40 while the volume fraction of nano particles $(\varphi)$ varied within the range of $0<\varphi<0.05$.

Comparison of the single and two-phase modeling for the nanofluids has been considered by the researchers. For instance, Fard et al. [16] compared the results of the single phase and two-phase numerical methods for nanofluids in a circular tube. They reported that for $\mathrm{Cu}$-water the average relative error between experimental data and the CFD results based on a single-phase model was $16 \%$ while for two-phase model was $8 \%$. In another numerical study, Göktepe et al. [17] compared these two models for nanofluid convection at the entrance of a uniformly heated tube. They found the same results and confirm the accuracy of two-phase modeling.

Since there is a lack of study in behavior of nanofluid around cylinder and due to its large application, the heat transfer from a circular cylinder with constant wall temperature is investigated by using numerical method.

\section{Governing equations and numerical method}

Consider the two-dimensional, laminar flow of an incompressible Newtonian fluid with a uniform inlet velocity $U_{\infty}$ and temperature $T_{\infty}$ across an infinitely long (in $z$-direction) circular cylinder (Fig. 1). To convert the physical problem into a computational equivalent, a circular cylinder of diameter $D$ is placed concentrically in a circular domain of diameter $D_{\infty}$. The radius of the enveloping circular domain is chosen to be sufficiently large in order to minimize the boundary effects. 


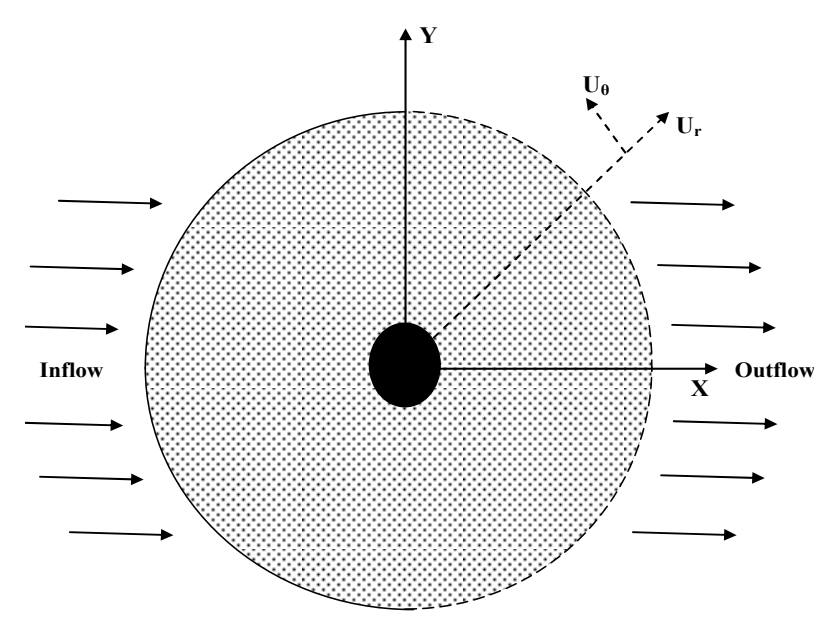

Figure 1. Schematic of computational domain and coordinate system.

The surface of the solid cylinder is maintained at a constant wall temperature, $\mathrm{T}_{\mathrm{w}}$. The thermo-physical properties of the streaming liquid are assumed to be independent of the temperature and the viscous dissipation effects in the energy equation are neglected in this study.

\subsection{Governing equations}

The flow and heat transfer phenomena are governed by the continuity, Navier-Stokes and thermal energy equations written in their dimensionless forms, as follows.

Continuity Equation:

$$
\frac{1}{r} \frac{\partial\left(r U_{\mathrm{r}}\right)}{\partial r}+\frac{1}{r} \frac{\partial U_{\theta}}{\partial \theta}=0
$$

$\theta$-Momentum Equation:

$$
\begin{aligned}
& \frac{\partial U_{\theta}}{\partial t}+\frac{U_{\theta}}{r} \frac{\partial U_{\theta}}{\partial \theta}+U_{\mathrm{r}} \frac{\partial U_{\theta}}{\partial r}+\frac{U_{\mathrm{r}} U_{\theta}}{r}= \\
& =-\frac{\rho_{\mathrm{f}}}{\rho_{\mathrm{nf}}} \frac{1}{r} \frac{\partial P}{\partial \theta}+\frac{2}{v_{\mathrm{f}} \operatorname{Re}} \frac{\mu_{\mathrm{nf}}}{\rho_{\mathrm{nf}}} . \\
& \cdot\left(\frac{1}{r} \frac{\partial}{\partial r}\left(r \frac{\partial U_{\theta}}{\partial r}\right)+\frac{1}{r^{2}} \frac{\partial^{2} U_{\theta}}{\partial \theta^{2}}+\frac{2}{r^{2}} \frac{\partial U_{\mathrm{r}}}{\partial \theta}-\frac{U_{\theta}}{r^{2}}\right)
\end{aligned}
$$

$$
\begin{aligned}
& \frac{\partial U_{\theta}}{\partial t}+\frac{U_{\theta}}{r} \frac{\partial U_{\theta}}{\partial \theta}+U_{\mathrm{r}} \frac{\partial U_{\theta}}{\partial r}+\frac{U_{\mathrm{r}} U_{\theta}}{r}= \\
& -\frac{\rho_{\mathrm{f}}}{\rho_{\mathrm{nf}}} \frac{1}{r} \frac{\partial P}{\partial \theta}+\frac{2}{v_{\mathrm{f}} \operatorname{Re}} \frac{\mu_{\mathrm{nf}}}{\rho_{\mathrm{nf}}} . \\
& \cdot\left(\frac{1}{r} \frac{\partial}{\partial r}\left(r \frac{\partial U_{\theta}}{\partial r}\right)+\frac{1}{r^{2}} \frac{\partial^{2} U_{\theta}}{\partial \theta^{2}}+\frac{2}{r^{2}} \frac{\partial U_{\mathrm{r}}}{\partial \theta}-\frac{U_{\theta}}{r^{2}}\right)
\end{aligned}
$$

$r$-Momentum Equation:

$$
\begin{aligned}
& \frac{\partial U_{\mathrm{r}}}{\partial t}+\frac{U_{\theta}}{r} \frac{\partial U_{\mathrm{r}}}{\partial \theta}+U_{\mathrm{r}} \frac{\partial U_{\mathrm{r}}}{\partial r}-\frac{U_{\theta}^{2}}{r}= \\
& =-\frac{\rho_{\mathrm{f}}}{\rho_{\mathrm{nf}}} \frac{\partial P}{\partial r}+\frac{2}{v_{\mathrm{f}} \operatorname{Re}} \frac{\mu_{\mathrm{nf}}}{\rho_{\mathrm{nf}}} . \\
& \cdot\left(\frac{1}{r} \frac{\partial}{\partial r}\left(r \frac{\partial U_{\mathrm{r}}}{\partial r}\right)+\frac{1}{r^{2}} \frac{\partial^{2} U_{\mathrm{r}}}{\partial \theta^{2}}-\frac{2}{r^{2}} \frac{\partial U_{\theta}}{\partial \theta}-\frac{U_{\mathrm{r}}}{r^{2}}\right)
\end{aligned}
$$

Energy Equation:

$$
\begin{aligned}
& \frac{\partial T}{\partial t}+\frac{U_{\theta}}{r} \frac{\partial T}{\partial \theta}+U_{\mathrm{r}} \frac{\partial T}{\partial r}= \\
& =\frac{k_{\mathrm{nf}}}{k_{\mathrm{f}}} \frac{\left(\rho C_{\mathrm{p}}\right)_{\mathrm{f}}}{\left(\rho C_{\mathrm{p}}\right)_{\mathrm{nf}}} \frac{2}{\operatorname{Re} \operatorname{Pr}} . \\
& \cdot\left(\frac{1}{r} \frac{\partial}{\partial r}\left(\frac{\partial T}{\partial r}\right)+\frac{1}{r} \frac{\partial}{\partial \theta}\left(\frac{1}{r} \frac{\partial T}{\partial \theta}\right)\right)
\end{aligned}
$$

\subsection{Thermal properties of nanofluids}

Density:

The density of nanofluids at different volume concentrations and temperatures are obtained from the literature. The nanofluids density calculated with the equation from Pak and Cho (1998).

$$
\rho_{\mathrm{nf}}=(1-\varphi) \rho_{\mathrm{f}}+\varphi \rho_{\mathrm{p}}
$$

For typical nanofluids with nano particles of volume fraction less than $1 \%$, a variation of less than $5 \%$ in the fluid density is expected.

Specific heat:

The specific heat of a nanofluid can be calculated by using energy balance as:

$$
\left(\rho C_{\mathrm{p}}\right)_{\mathrm{nf}}=(1-\varphi)\left(\rho C_{\mathrm{p}}\right)_{\mathrm{f}}+\varphi\left(\rho C_{\mathrm{p}}\right)_{\mathrm{p}}
$$


Where, $\varphi$ is the nano particle volume fraction and is given as:

$$
\varphi=\frac{\text { Volume of nanoparticles }}{\text { Total volume of solution }}
$$

The Eqs. (6) and (7) were introduced by Buongiorno (2006).

The Brownian motion has a significant impact on the effective thermal conductivity. Koo and Kleinstreuer (2005) proposed that the effective thermal conductivity is composed of the particle's conventional static part and a Brownian motion part. This 2-component thermal conductivity model takes into account the effects of particle size, particle volume fraction, and temperature.

$$
\begin{gathered}
k_{\mathrm{nf}}=k_{\text {static }}+k_{\text {Brownian }} \\
\frac{k_{\text {static }}}{k_{\mathrm{f}}}=\frac{k_{\mathrm{p}}+2 k_{\mathrm{f}}-2\left(k_{\mathrm{f}}-k_{\mathrm{p}}\right) \varphi}{k_{\mathrm{p}}+2 k_{\mathrm{f}}+\left(k_{\mathrm{f}}+k_{\mathrm{p}}\right) \varphi} \\
k_{\text {Brownian }}=5 \times 10^{4} \beta \varphi \rho_{\mathrm{f}} C_{\mathrm{p} . \mathrm{f}} \sqrt{\frac{k T}{2 \rho_{\mathrm{p}} d_{\mathrm{p}}} f(T, \varphi)}
\end{gathered}
$$

Where $k=1.3809 \times 10^{-23} \mathrm{~J} / \mathrm{K}$ is the Boltzmann constant, and $\beta$ is given as:

$$
\beta=8.4407(100 \varphi)^{-1.07304}
$$

and $f(T, \varphi)$ is given as:

$$
\begin{aligned}
& f(T, \varphi)=\left(2.8217 \times 10^{-2} \varphi+3.917 \times 10^{-3}\right)\left(\frac{T}{T_{0}}\right) . \\
& \cdot\left(-3.0669 \times 10^{-2} \varphi-3.91123 \times 10^{-3}\right)
\end{aligned}
$$

The effective dynamic viscosity for the nano-fluid could be calculated by the following equations (Corcione 2010):

$$
\begin{gathered}
\mu_{\mathrm{nf}}=\frac{\mu_{\mathrm{f}}}{\left(1-34.87\left(d_{\mathrm{p}} / d_{\mathrm{f}}\right)^{-0.3} \times \varphi^{1.03}\right)} \\
d_{\mathrm{f}}=\left(\frac{6 M}{N \pi \rho_{\mathrm{f} 0}}\right)^{1 / 3}
\end{gathered}
$$

where $d_{\mathrm{p}}$ and $d_{\mathrm{f}}$ represented the mean diameter of the nanoparticles and equivalent diameter of a base fluid molecule, respectively; $M$ represented the molecular weight; $N$ represented the Avogadro number $=$ $6.022910^{23} \mathrm{~mol}^{-1}$; and $\rho_{\mathrm{fo}}$ is the density of the base fluid found at temperature $=293 \mathrm{~K}$.

\subsection{Boundary conditions}

According to Fig. 1 the governing Eqs. (1) - (5) are subjected to the following boundary conditions: Inlet boundary $\left(r=R_{\infty}=40 R,-\pi / 2 \leq \theta \leq \pi / 2\right)$ :

$$
U_{\theta}=\sin \theta, U_{\mathrm{r}}=-\cos \theta, T=T_{\infty}
$$

Outlet boundary $\left(r=R_{\infty}=40 R, \pi / 2 \leq \theta \leq-\pi / 2\right)$ :

$$
\frac{\partial U_{\theta}}{\partial r}=0, \frac{\partial U_{\mathrm{r}}}{\partial r}=0, \frac{\partial T}{\partial r}=0
$$

Cylinder wall boundary $(r=R, 0 \leq \theta \leq 2 \pi)$ :

$$
U_{\theta}=0, U_{\mathrm{r}}=0, T=T_{\mathrm{w}}
$$

\subsection{Auxiliary equations:}

- The wall pressure coefficient, $C_{\mathrm{P}}$, may be defined as the following:

$$
C p=\frac{P-P_{0}+0.5 \rho U_{\infty}^{2}}{0.5 \rho U_{\infty}^{2}}
$$

where, $P_{0}$ is the pressure at the front stagnation point.

- In the time-periodic flow regime, there is also a net force acting on the cylinder in the lateral direction and this is expressed in terms of a lift coefficient $\mathrm{C}_{\mathrm{L}}$, defined as follows:

$$
C_{\mathrm{L}}=\frac{F_{\mathrm{L}}}{0.5 \rho D U_{\infty}^{2}}
$$

where $F_{\mathrm{L}}$ is the lift force.

- The local Nusselt number of the nanofluid, based on cylinder diameter is defined as:

$$
N u=-\left[\frac{k_{\mathrm{nf}}}{k_{\mathrm{f}}} \frac{\partial \theta}{\partial n}\right]_{\text {along the cylinder surface }}
$$


- Surface averaged Nusselt number of fully developed thermal boundary layer is defined as:

$$
N u_{\mathrm{ave}}=\frac{1}{s} \int_{s} N u d s
$$

\subsection{Grid generation}

In the present meshing scheme, cylinder with a diameter $D$ resides in the center of the chosen computational domain. The outer boundary of the domain is circular with diameter $D_{\infty}$ from the center of the cylinder, Fig. 1. Number of node points along the cylinder circumference and along the normal direction is represented by ' $\mathrm{N}$ ' and ' $\mathrm{M}$ ' respectively. We use $M=144$ nodes stretched along the radial direction and $\mathrm{N}=121$ equispaced nodes around the circumferential direction. The wall normal distance of the first internal grid point is maintained at $\Delta r=0.0001 \mathrm{D}$, required for adequate resolution of the sharp near wall gradients of the flow variables.

\subsection{Numerical Method}

The present numerical investigation has been carried out using FLUENT (version 6.3.26). The unstructured quadrilateral cells of non-uniform grid spacing are generated using the commercial software GAMBIT (version 2.3.16). The grid is chosen to be sufficiently fine to capture the steep gradients in the vicinity of the cylinder. The second order upwind scheme has been used to discretize the convective terms in the momentum and energy equations. The SIMPLE scheme (semi implicit method for the pressure linked equations) is used for solving the pressure-velocity decoupling. FLUENT solves the system of algebraic equations using the Gauss-Siedel (G-S) point-by-point iterative method.

The thermos-physical properties of the fluid and the nanoparticle, copper, are listed in Table 1.

Table 1. Thermophysical properties of nanoparticle and base fluids

\begin{tabular}{|c|c|c|c|c|}
\cline { 2 - 5 } \multicolumn{1}{c|}{} & $\begin{array}{c}k \\
{[\mathrm{~W} / \mathrm{mK}]}\end{array}$ & $\begin{array}{c}\rho \\
{\left[\mathrm{Kg} / \mathrm{m}^{3}\right]}\end{array}$ & $\begin{array}{c}\mu 10^{-3} \\
{[\mathrm{mPa} . \mathrm{S}]}\end{array}$ & $\begin{array}{c}C_{p} \\
{[\mathrm{~J} / \mathrm{kgK}]}\end{array}$ \\
\hline \hline $\begin{array}{c}\text { Water } \\
\mathrm{Cu}\end{array}$ & $\begin{array}{c}0.613 \\
400\end{array}$ & $\begin{array}{c}997.1 \\
8954.0\end{array}$ & $\begin{array}{c}1.003 \\
\cdots \cdots\end{array}$ & $\begin{array}{c}4179.0 \\
383.0\end{array}$ \\
\hline
\end{tabular}

\section{Results and discussion}

\subsection{Validation of results}

In order to validate our numerical solution, estimated results for drag coefficients in forced convective heat transfer of clear fluid flow around a circular cylinder are compared with the available data in the literature. The separate runs were necessary to determine the results at specific Reynolds numbers of 100 and 200. Table 2 compares the drag coefficient $C_{\mathrm{D}}$ obtained in the present research with those from references [218-19-20-21-22]. It is clear that the results are in very good agreement with the previous studies.

Table 2. Comparison between the present results and literature data for at Re 100 and 200

\begin{tabular}{|lll||ll|}
\hline Refrence & $\mathbf{R e}=$ & $\mathbf{1 0 0}$ & $\mathbf{R e}=$ & $\mathbf{2 0 0}$ \\
\cline { 2 - 5 } & $C_{\mathrm{D}}$ & $\mathrm{St}$ & $C_{\mathrm{D}}$ & $\mathrm{St}$ \\
\hline Ha. Roy [20] & 1.352 & 0.161 & 1.32 & 0.192 \\
I. , M. S. [19] & 1.344 & 0.165 & 1.337 & 0.194 \\
Ding et al. [18] & 1.356 & 0.166 & 1.348 & 0.196 \\
B.N. Ra. [2] & 1.368 & 0.172 & 1.376 & 0.192 \\
Lam et al. [21] & 1.36 & 0.160 & 1.32 & 0.196 \\
M. and A. [22] & 1.368 & 0.172 & 1.376 & 0.192 \\
Present study & 1.322 & 0.163 & 1.316 & 0.195 \\
\hline
\end{tabular}

Fig. 2 shows the temporal evolution of the lift and drag coefficients computed for Reynolds number $\mathrm{Re}=100$. The differences observed between the base fluid and nanofluid. The magnitude of the maximum lift coefficient and the mean drag coefficient are, in general, observed to be less for the base fluid.

\subsection{Fluid flow}

Fig. 3 shows the distribution of pressure coefficient for various solid volume fractions at the surface of cylinder. As shown in Fig.3, in favorable pressure gradient region, the value of $C_{\mathrm{p}}$ decreases for different solid volume fractions, however it increases in adverse pressure gradient region. At the accelerating flow zone covering the front part of the cylinder, the value of $C_{\mathrm{p}}$ is independent of volume fraction number. Its value approached to constant value of 1 for all values of $\varphi$.

\subsection{Heat transfer}

A comparison between local Nusselt number on the surface of cylinder for various Reynolds numbers and different solid volume fractions is shown in Fig. 4. This figures indicate that any increase in the solid 
concentration $(\varphi)$ improves the effective thermal

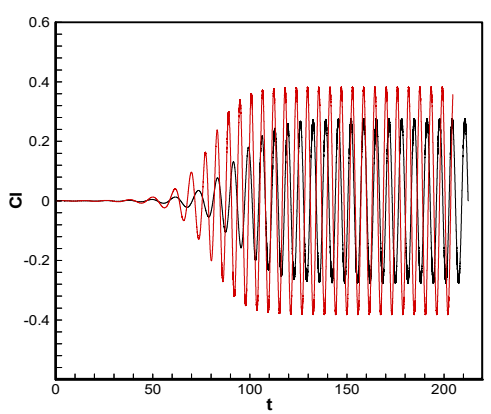

conductivity and consequently the rate of heat transfer.

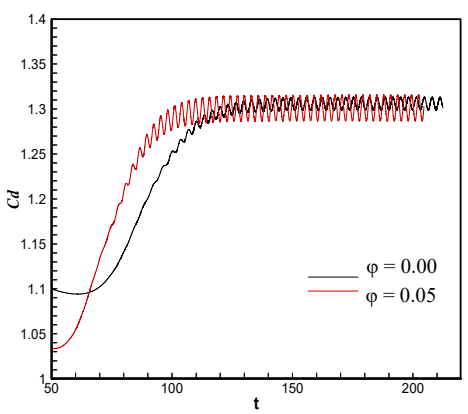

Figure 2. Temporal evolution of lift and drag coefficients for laminar flow past a circular cylinder at Re $=100$.

a)

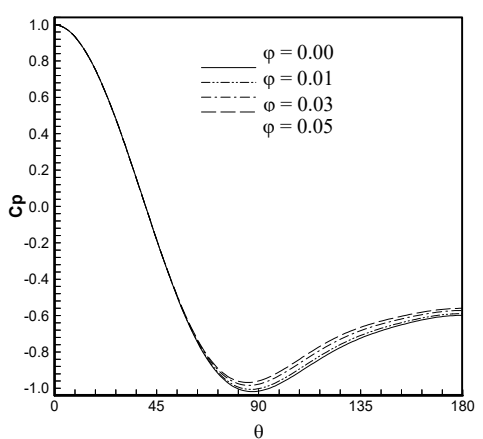

b)

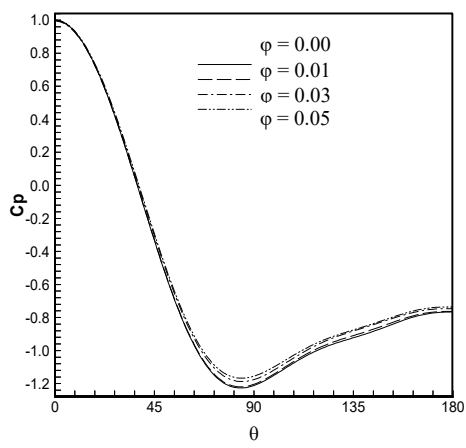

Figure 3. Distribution of pressure coefficient around surface of cylinder for various solid volume fractions $\varphi$ at a) $R e=50$, b) $R e=100$.

a)

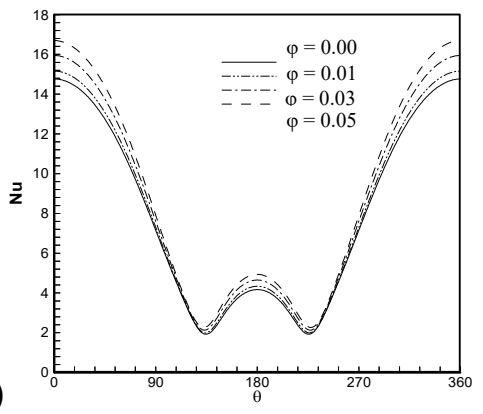

c)

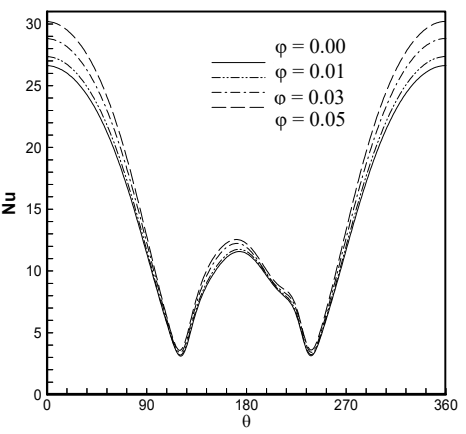

b)

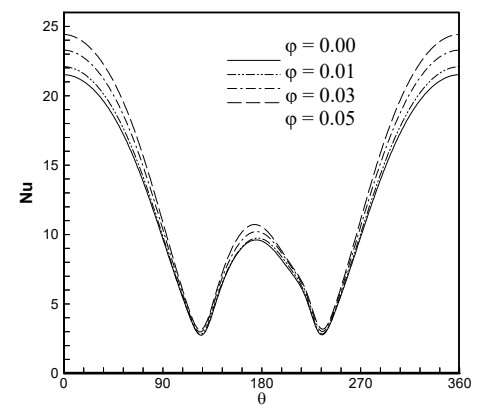

d)

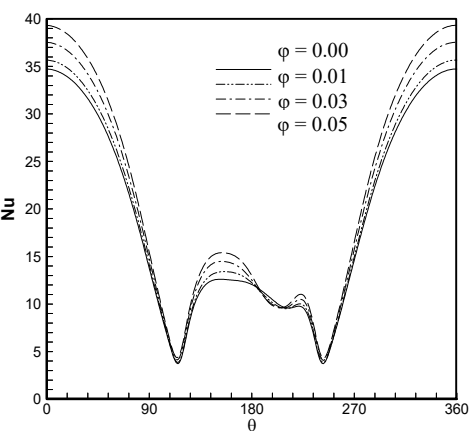

Figure 4. Variation of local Nusselt number on cylinder surface with various solid volume fraction $\varphi$ at a) $\operatorname{Re}=50$, b) $\operatorname{Re}=100, c) \operatorname{Re}=200$, d) $\operatorname{Re}=300$ 
Also, thermal boundary layer is decreased by any increase in solid volume fraction. It shows that local Nusselt number is enhanced by adding nanoparticles to the base fluid. The data show an increase of up to $20 \%$ in convection heat transfer as the particle volume fraction is increased to $3 \%$.

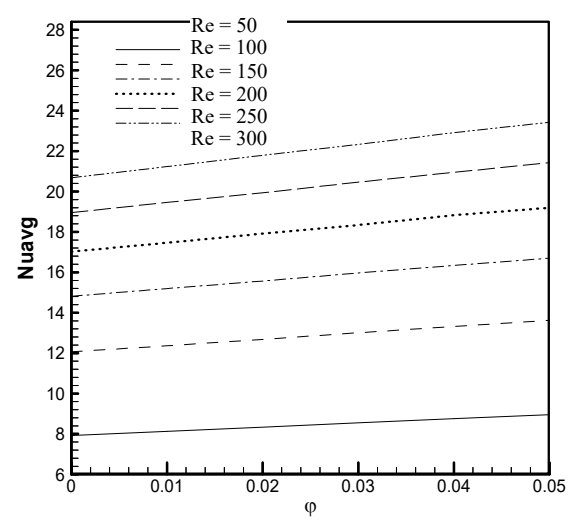

Figure 5. Variation of average Nusselt number on the wall of cylinder versus solid volume fractions, $\varphi$ for different Re.

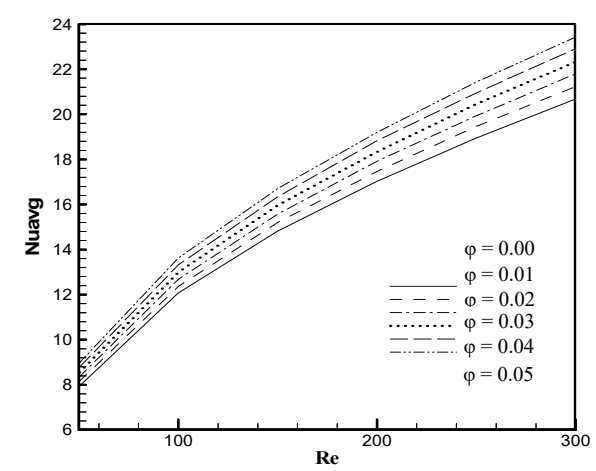

Figure 6. Variation of average Nusselt number on the wall of cylinder versus Reynolds number for different solid volume fractions, $\varphi$.

The variation of average Nusselt number for different values of Reynolds number and solid fraction is shown in Fig. 5 and Fig. 6, respectively. In these figures, we show that an increase in both Reynolds numbers and solid volume fraction will increase the average Nusselt number.

Fig. 7 illustrated the streamlines, vorticity and isotherm contours around the cylinder, for Reynolds numbers of 100 and 200. Here, the contours of base fluid and nanofluid are shown for comparison. Here, red lines are used for nanofluid and black solid lines are used for the base fluid. This figure indicated that the magnitude of the maximum negative velocity in recirculation zone is increased by any increment in solid concentration and Reynolds number. In the base fluid, the strength of the vorticity is increased in comparison with the nanofluid.

For the temperature distribution contours, it can be concluded that the temperature contours are steeper in the near-wake region with increasing Reynolds number. This signifies that higher Reynolds number sets a higher temperature gradient, leading to an enhanced heat transfer from the cylinder. Thus, due to higher temperature gradient, temperature contours are much denser near the front surface of the cylinder. It can also be seen that the nanofluid show higher heat transfer rate from the cylinder than base fluid.

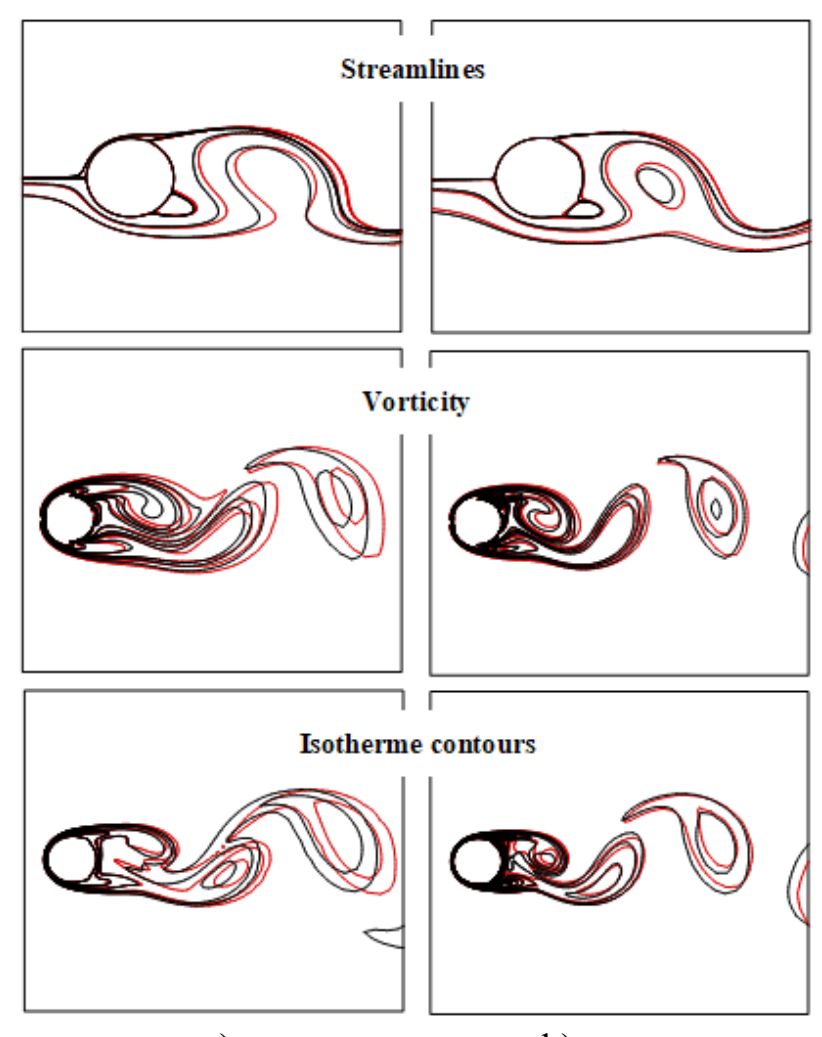

a)

b)

Figure 7. The streamlines, vorticity and isotherm contours around the cylinder, (base fluid (black lines) and water $/ \mathrm{Cu} 5 \%$ (red lines) at a) $\operatorname{Re}=100$, b) $\operatorname{Re}=200$.

\section{Conclusion}

Single models have been investigated for the characterization of laminar forced convection of $\mathrm{Cu}$ water nanofluid with various concentrations around a 
circular cylinder. In this article, the point of research was to evaluate the effect of nano-particle on convective heat transfer and flow characteristics. The significant observations made on the forced convection around a circular cylinder are summarized as follows:

1) The vorticity, pressure coefficient, and recirculation length are increased by the addition of nanoparticles into the base fluid.

2) The Local Nusselt number, average Nusselt number, and heat transfer coefficient of a nanofluid is augmented by increasing the volume fraction of nanoparticles.

3) The temperature gradient at the cylinder surface along normal direction drops with increase in nano particle concentration. However, there is an increase in the thermal conductivity of nanofluid, thus leading to increase in Nusselt number.

$\begin{array}{ll}\text { Nomenclature } \\ C_{\mathrm{D}} \quad \text { coefficient of drag } \\ C_{\mathrm{L}} & \text { coefficient of lift } \\ D & \text { cylinder diameter, } \mathrm{m} \\ k & \text { thermal conductivity, } \mathrm{W} / \mathrm{m} \mathrm{K} \\ P & \text { non-dimensional pressure } \\ \mathrm{Pr} & \text { Prandtl number, } \mathrm{Pr}=\mathrm{v} / \alpha \\ \mathrm{Re} & \text { Reynolds number, } R e=\rho U_{\infty} D / \mu \\ t & \text { non-dimensional time } \\ T & \text { non- dimensional temperature } \\ U_{\mathrm{r},} U_{\theta} & \text { non-dimensional velocity components } \\ r, \theta & \text { non-dimensional coordinates }\end{array}$

\section{Greek}

$\begin{array}{ll}\mu & \text { viscosity } \\ \rho & \text { density } \\ v & \text { kinetic viscosity }\end{array}$

\begin{tabular}{ll}
\multicolumn{2}{l}{ Subscripts } \\
$\infty$ & inlet condition \\
$\mathrm{w}$ & wall \\
avg & average \\
$\mathrm{f}$ & fluid \\
$\mathrm{p}$ & solid \\
$\mathrm{nf}$ & nanofluid
\end{tabular}

\section{References}

[1] Roshko, A.: On the development of turbulent wakes from vortex streets, Technical Report No. NACA TR 1191 (California Institute of Technology, 1953).
[2] Rajani, B.,N., Kandasamy, A., Majumdar, S.: Numerical simulation of laminar flow past a circular cylinder, Appl. Math. Modelling 33, (2009) 1228-1247.

[3] Norberg, C.: An experimental investigation of the flow around a circular cylinder: influence of aspect ratio, J. Fluid Mech. 258, (1994) 287316.

[4] Mahfouz, F. M., and Badr, H .M.: Forced convection from a rotationally oscillating cylinder placed in a uniform stream, Int. J. of Heat and Mass Transfer, 43, (2000), 3093-3104.

[5] Taneda S.: Visual observations of the flow past a circular cylinder performing a rotatory oscillation, J. Phys. Soc. Jpn. 45 (1978), 1038 1043.

[6] Williamson, C. H. K.: Oblique and parallel modes of vortex shedding in the wake of a circular cylinder at low Reynolds numbers, J. of Fluid Mech., 206 (1989), 579-627.

[7] Masuda, H., Ebata, A., Teramea, K., Hishinuma, N.: Altering the thermal conductivity and viscosity of liquid by dispersing ultra-fine particles, Netsu Bussei, 4 (1993), 227-233.

[8] Pak, B.C., Cho, Y.I.: Hydrodynamic and heat transfer study of dispersed fluids with submicron metallic oxide particles, Exp. Heat Transfer, 11 (1998), 151-170.

[9] Xuan, Y., Li, Q., Investigation on Convective Heat Transfer and Flow Features of Nanofluids, J. of Heat Transfer, 125 (2003), 151-155.

[10] Mintsa, H. A., Roy, G., Nguyen, C. T., Doucet, D.: New temperature dependent thermal conductivity data for water-based nanofluids, Int. J. Thermal Sciences, 48 (2009), 363-371.

[11] Dalkilic, A. S., Kayaci, N., Celen, A., Tabatabaei, M., Yildiz, O., Daungthongsuk, W., Wongwises, S.: Forced Convective Heat Transfer of Nanofluids - A Review of the Recent Literature, Curr. Nanosci, 8 (2012), 949-969.

[12] Sarkar, S., Ganguly, S., Dalal, A.: Buoyancy driven flow and heat transfer of nanofluids past a square cylinder in vertically upward flow, Int J Heat Mass Transfer, 59 (2013), 433-450.

[13] Farooji, V. E., Bajestan, E. E., Niazmand, H., Wongwises, S.: Unconfined laminar nanofluid flow and heat transfer around a square cylinder, Int J Heat Mass Transf, 55 (2012), 1475-1485.

[14] Valipour, M.S., Ghadi, A.Z.: Numerical investigation of fluid flow and heat transfer around a solid circular cylinder utilizing nanofluid, Int Comm. Heat and Mass Transfer, 38 (2011), 1296-1304. 
[15] Valipour, M. S., Masoodi, R., Rashidi, S., Bovand, M., Mirhosseini, M.: A numerical study on convection around a square cylinder using Al2O3-H2O nanofluid, Therm. Sci, 18 (2014) 1305-1314.

[16] Fard, M. H., Esfahany, M. N., Talaie, M. R.: Numerical study of convective heat transfer of nanofluids in a circular tube two-phase model versus single-phase model, Int. Comm. Heat Mass Transfer, 37 (2010), 91-97.

[17] Göktepe, S., Atalik, K., Ertürk, H.: Comparison of single and two-phase models for nanofluid convection at the entrance of a uniformly heated tube, Int J Therm. Sci, 80 (2014), 83-92.

[18] Ding, H., Shu, C., Yeo, K.S., Xu, D.: Numerical simulation of flows around two circular cylinders by mesh-free least square-based finite difference methods. Int. J. for Num. Meth. in Fluids, 53 (2007), 305-332.

[19] Harimi, I., Saghafian, M.: Numerical simulation offluid flow and force d convection heat transfer from tandem circul ar cylinders using overset grid method, J. of Fluids and Structures, 28 (2012), $309-327$.

[20] Harichandan, A.B., Roy, A.: Numerical investigation of low Reynolds number flow past two and three circular cylinders using unstructured grid CFR scheme, Int. J. of Heat and Fluid Flow 31, 154-171.

[21] Lam, K., Gong, W.Q., So, R.M.C.: Numerical simulation of cross-flow around four cylinders in an in-line square configuration, J. of Fluids and Structures, 24 (2008), 34-57.

[22] Mahir, N., Altac, Z.: Numerical investigation of convective heat transfer in unsteady flow past two cylinders in tandem arrangements. I. J. of Heat and Fluid Flow, 29 (2008), 1309-1318.
[23] Xuan, Y., Li, Q.: Investigation on Convective Heat Transfer and Flow Features of Nanofluids, ASME J. Heat Transfer, 125, (2003) 151-155.

[24] Mintsa, H. A., Roy, G., Nguyen, C.T., Doucet, D.: New temperature dependent thermal conductivity data for water-based nanofluids, Int. J. Thermal Sciences, 48 (2009), 363-371.

[25] Norberg, C.: An experimental investigation of the flow around a circular cylinder: influence of aspect ratio, Journal of Fluids Mechanics 1994; 258, 287-316.

[26] Maiga, S. E. B., Nguyen, C. T., Galanis, N., Roy G.: Heat transfer behaviours of nanofluids in a uniformly heated tube, Superlattices Microstruct, 35, (2004) 543 - 557.

[27] Pak, B. C., Cho, Y. I., Hydrodynamic and heat transfer study of dispersed fluids with submicron metallic oxide particles, Exp. Heat Transf., 11 (1998), 151 - 170.

[28] Hamilton, R. L., Crosser, O. K.: Thermal conductivity of heterogeneous 2 component systems, Ind. Eng. Chem. Fund. 1 (1962) 187.

[29] Brinkman, H. C., The viscosity of concentrated suspensions and solution, J. Chem. Phys. 20 (1952), 571-581.

[30] Khelili, Y., Allali, A., Bouakkaz, R.: Convective heat transfer characteristics of low Reynolds number nanofluid flow around a circular cylinder, M. and Materials Engineering, 23 (2017), 83-97.

[31] Bouakkaz, R., Salhi, F., Khelili, Y., Ouazzazi M. and Talbi, K.: Unconfined laminar nanofluid flow and heat transfer around a rotating circular cylinder in the steady regime, Arch. of thermod., 38 (2017), 3-20. 